\title{
The Feasibility of Waterproof Microcapsule System for Bacteria-Based Self-Healing Cementitious Material
}

\author{
Bing Liu, Biqin Dong, Ningxu Han, and Fing Xing \\ Guangdong Provincial Key Laboratory of Durability for Marine Civil Engineering, Shenzhen Durability Center for \\ Civil Engineering, College of Civil Engineering, Shenzhen University, China \\ Bing Liu \\ School of Transportation and Environmental Science, Shenzhen Institute of Information Technology, China \\ Xu Deng \\ College of Life Science, Shenzhen Key Laboratory of Marine Bioresource and Eco-Environmental Science, \\ Shenzhen University, China \\ Guangmin Zhu \\ Shenzhen Key Laboratory of Special Functional Materials, College of Materials Science and Engineering, \\ Shenzhen University, China
}

\begin{abstract}
In this study, a waterproof material was used to fabricate microcapsule by interfacial curing reaction to encapsulate an alkaliphilic spore-forming bacterium. The technical feasibility of encapsulated spores and the influence of three kinds of curing agent on the calcium precipitation activity (CPA) of the bacterium were studied. Furthermore, micromorphology of microcapsules was observed by Scanning Electron Microscopy (SEM). Afterwards, the thermal stability and thermolysis temperature were determined by TGA thermal analyzer. Moreover, the CPA of broken/ unbroken microcapsules was evaluated. In addition, water resistance was evaluated by adding microcapsules in the water for 1, 3, 7, 14, 28, and 56 days. Finally, light microscope was applied to trace the self-healing behavior of encapsulated mineralization bacterium in cement paste specimens.

The results showed that compared with unbroken microcapsules, higher CPA was achieved by breaking the microcapsule to release the bacterium, suggesting good protection for the encapsulated spores. Three curing agents showed nearly similar influence on the spores, while KH792 performed relatively better, and thus was used to fabricate microcapsule with the core/shell weight ratio being 1:1. Our results also indicated that ER microcapsules could keep unbroken in the water for 2 months. Compared with the specimens without embedded bacterium, the healed crack area of specimens embedded with bacterial microcapsules was monitored, suggesting effective self-healing of concrete crack can be achieved by introducing encapsulated mineralization microorganisms into concrete structures. Therefore, we put forward that this waterproof epoxy resin microcapsules could be potential for the application of self-healing concrete.
\end{abstract}

\section{INTRODUCTION}

In recent years, the development of self-healing technique in concrete based on microbial calcium carbonate precipitation has been becoming more and more promising. However, it is necessary to set up a waterproof protection strategy for embedded self-healing bacteria in the concrete during casting, hydration period, and service life before cracks occur. Epoxy resin has been widely used in concrete as the crack repairing agent, but so far no microcapsule was made from epoxy resin to encapsulate bacteria for concrete self-healing purpose.

\section{MATERIALS AND METHODS}

\subsection{Preparation of microcapsules}

Under the condition of double oil phase, suspension dispersion method was applied to prepare the waterproof biomicrocapsule. Microcapsules were fabricated by using a waterproof material, epoxy resin (ER), and an alkaliphilic spore-forming bacterium, Bacillus pseudofirmus DSM8715.

Trimethoxy silane [3-(2-aminoethyl)aminopropyl] was employed as silane coupling agent $\mathrm{KH} 792$. The influence factors on DSM8715 spore production and activity, such as the optimum $\mathrm{pH}$ value to induce 
mineralization, the optimum germination agent, the optimum buffer, the influence of three kinds of curing agent (silane coupling agent KH792, DMP-30, and $\mathrm{m}$-Xylylenediamine), which were used as curing agent for the fabrication of microcapsule, were studied by monitoring the calcium precipitation activity (CPA) of the bacterium before encapsulation.

In addition, microcrystalline cellulose (MCC), which was used as filling skeleton, was mixed with spore to prepare the core material. The mixture was formed into pellets with a cold extrusion rounded pelletizing machine. In order to obtain a suitable curing for the shell of microcapsule, silane coupling agent KH792 was used. Suspension dispersion process was carried out to form the outer coating of the microcapsules.

\subsection{Characterization of the microcapsules}

Micromorphology of microcapsules was observed by Scanning Electron Microscopy (SEM). Afterwards, the thermal stability and thermolysis temperature were determined by TGA thermal analyzer. Moreover, the CPA of broken/unbroken microcapsules was evaluated. In addition, water resistance was evaluated by adding microcapsules in the water for $0 \mathrm{~h}, 15$ days, and 30 days.

\subsection{Healing process}

Bacterial spores were encapsulated, and then microcapsules were introduced into cement paste specimens during mixture period. After 14 days of hydration period, the specimens were subjected to a splitting test to make crack. The crack closure behavior of the specimens with/without biomicrocapsules was studied quantitatively by light microscopy. Furthermore, efficiency of self-healing performance was evaluated with healed crack width.

\section{RESULTS AND DISCUSSION}

About spores, our experiment results showed that DSM8715 had a strong mineralization ability and grew fast at $30^{\circ} \mathrm{C}, \mathrm{pH} 9.5-11$. The influence factors on DSM8715 spore production were evaluated. The results showed that the optimum germination agent is inosine with the suitable concentration of $80 \mathrm{mmol} / \mathrm{L}$; the optimum buffer is $N$-cyclohexyl-3amino propylsulfonic acid (CAPS); the optimum $\mathrm{pH}$ for DSM8715 to induce mineralization was 10 with spore mineralization activity being $86.8 \%$ (Figure 1 ).

Three curing agents showed nearly similar influence on the spores, while $\mathrm{KH} 792$ performed relatively better, and thus was used to fabricate microcapsule (Figure 2). The microcapsule has a very good spherical shape, and the size is about $500 \mu \mathrm{m}$ in diameter. The shell is smooth and compact with the thickness of about $50 \mu \mathrm{m}$ and the core/shell weight ratio of 1:1 (Figure 3). Compared with unbroken microcapsules, the results showed that higher CPA was achieved by breaking the microcapsule to release the bacterium, suggesting good protection for the encapsulated spores (Figure 4). Our results also indicated that ER microcapsules could keep unbroken in the water for 56 days, as there were no any changes in shape and size (Figure 5).

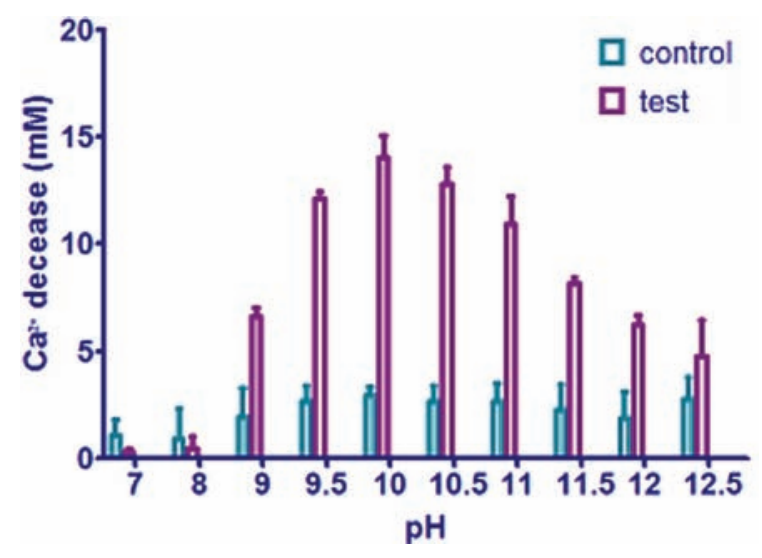

Figure 1. The influence of $\mathrm{pH}$ on the bacterial mineralization.

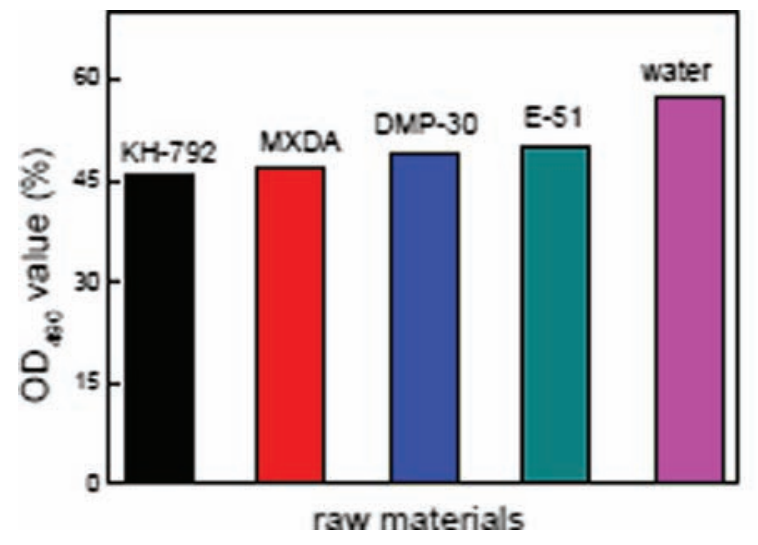

Figure 2. The influence of organic solvents on the bacterial calcium precipitation.

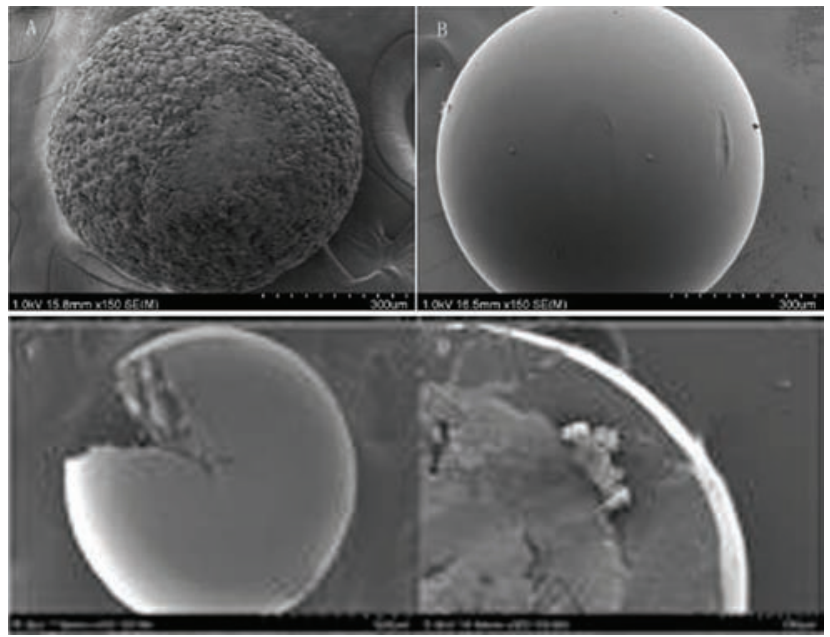

Figure 3. The SEM images of microcapsule before and after encapsulation. 


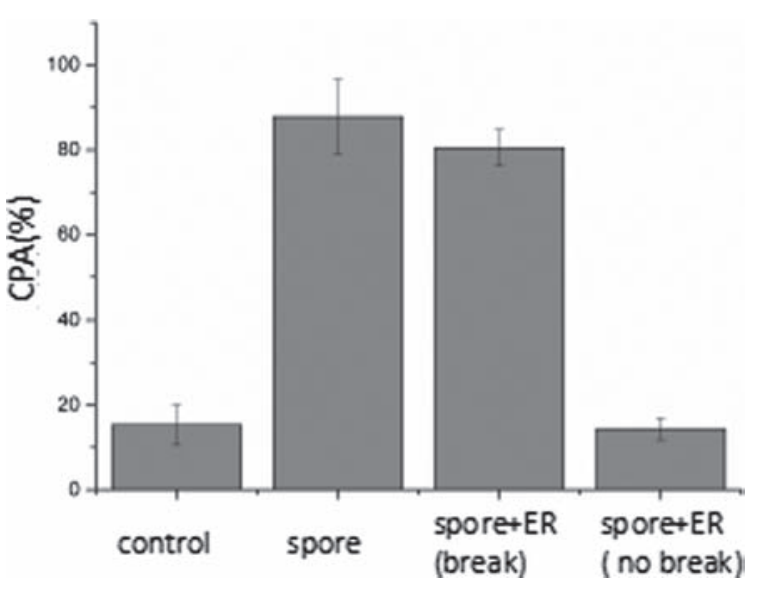

Figure 4. $\mathrm{CPA}$ of bacteria in non- $\mathrm{CO}_{2}$ incubation system.

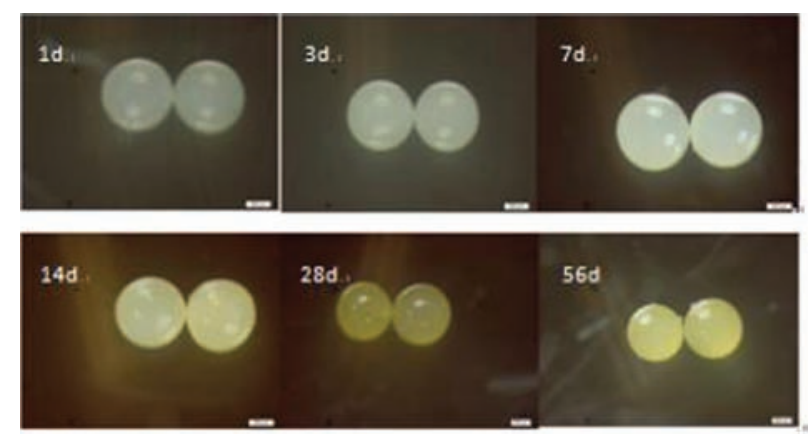

Figure 5. Micrograph of waterproof process of ER microcapsules after $1,3,7,14,28$, and 56 days.

The crack-healing process, mechanism, and healing effectiveness were studied to evaluate the feasibility of self-healing system. Compared with the specimens without embedded bacterium, the healed crack width of specimens embedded with bacterial microcapsules was higher and faster (Figure 6), suggesting effective self-healing of concrete crack can be achieved by introducing encapsulated mineralization microorganisms into concrete structures. Therefore, we put forward that this waterproof epoxy resin microcapsules could be potential for the application of self-healing concrete.

\section{CONCLUSION}

In this study, a microcapsule-based microbial selfhealing system with epoxy resin and an alkaliphilic spore-forming bacterium was designed and fabricated, and the physical properties and morphology of spore and microcapsules were evaluated for self-healing system. It can be concluded that the microcapsules have excellent waterproof performance and the adopted waterproof biomicrocapsule-based selfhealing system is suitable to fulfill the healing functionality in cementitious materials.

\section{ACKNOWLEDGMENTS}

The authors would like to acknowledge financial support provided by National Natural Science Foundation of China (No. 51120185002, 51508338), Scientific Research Foundation for Returned Scholars, Ministry of Education of China (20151098), Guangdong Natural Science Foundation (2015A030310128), and Shenzhen R\&D Fund (JCYJ20140418091413530).

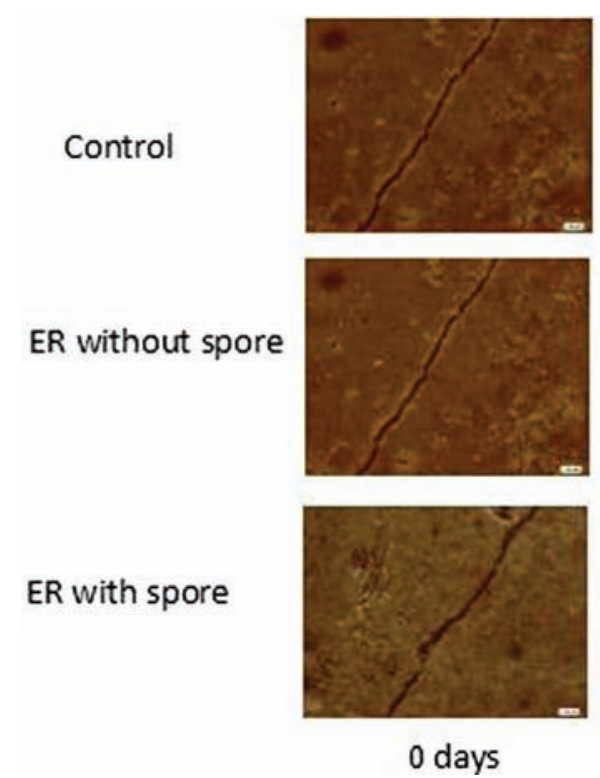

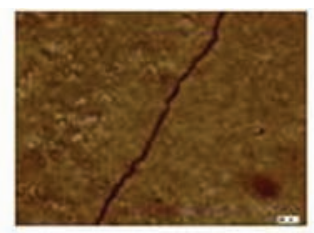
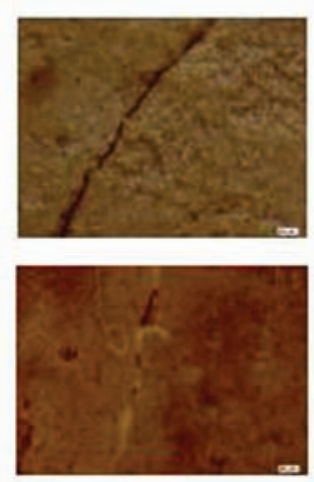

10 days
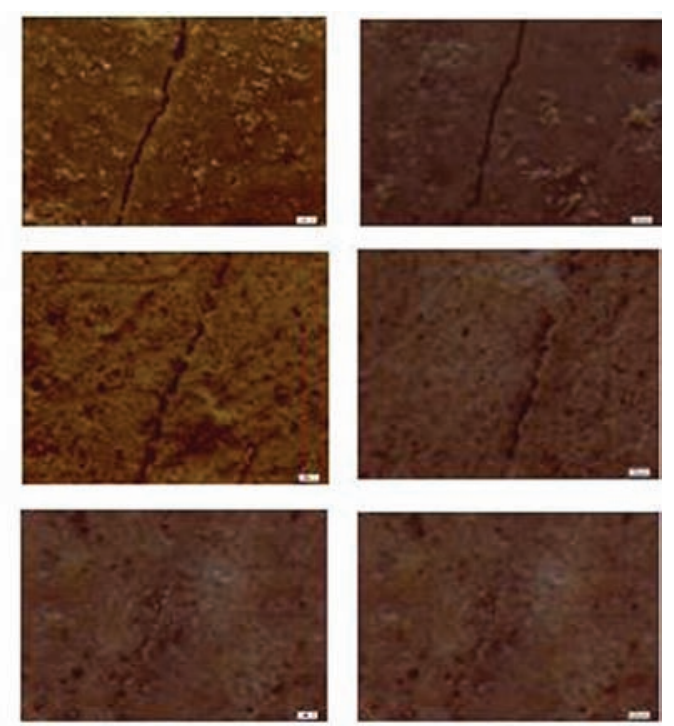

20 days

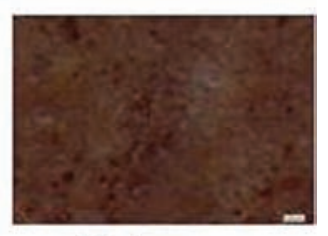

30 days

Figure 6. Breakage of crack and subsequent healing procedure was visually monitored by light microscope. 


\section{REFERENCES}

De Muynck, W., De Belie, N., \& Verstraete, W. (2010). Microbial carbonate precipitation in construction materials: A review. Ecological Engineering, 36(2), 118-136.

Jonkers, H. M., Thijssen, A., Muyzer, G., Copuroglu, O., \& Schlangen, E. (2010). Application of bacteria as self-healing agent for the development of sustainable concrete. Ecological Engineering, 36(2), 230-235.
Van Tittelboom, K., \& De Belie, N. (2013). Selfhealing in cementitious materials - A review. Materials, 6(6), 2182-2217.

Wang, J. Y., Soens, H., Verstraete, W., \& De Belie, N. (2014). Self-healing concrete by use of microencapsulated bacterial spores. Cement and Concrete Research, 56, 139-152.

Xu, J., Yao, W., \& Jiang, Z. (2013). Non-ureolytic bacterial carbonate precipitation as a surface treatment strategy on cementitious materials. Journal of Materials in Civil Engineering, 26(5), 983-991. 